\title{
MultiTela GD: um recurso para a formação de professores de matemática em EAD.
}

\author{
Vandoir Stormowski - PUCRS - vandoir@yahoo.com.br \\ Maria Alice Gravina - UFRGS - gravina@mat.ufrgs.br \\ José Valdeni de Lima - UFRGS - valdeni@inf.ufrgs.br
}

\begin{abstract}
Resumo: este artigo tem o objetivo de apresentar o recurso MultiTela GD, que foi desenvolvido para atender as necessidades da formação de professores de matemática na modalidade EAD, para o uso de softwares de Geometria Dinâmica (GD). São apresentadas as motivações para o seu desenvolvimento, bem como uma descrição detalhada de cada parte que compõe a MultiTela. O recurso é desenvolvido a partir do estudo dos Registros de Representação Semiótica, aporte teórico importante para o processo de aprendizagem em matemática, bem como da perspectiva de explorar adequadamente o Potencial Semiótico dos softwares de GD. Possuem destaque as implicações destes aportes teóricos no desenvolvimento da MultiTela GD. É apresentada uma breve descrição de sua implementação em curso de formação de professores de matemática na modalidade EAD, e da sua receptividade pelos participantes.
\end{abstract}

Palavras-chave: MultiTela; geometria dinâmica; educação a distância; professores de matemática.

\section{MultiTela GD: a resource for the training of mathematics teachers in Distance Education.}

\begin{abstract}
This article aims to present the MultiTela GD feature, which was developed to meet the training needs of mathematics teachers in distance education, for the use of Dynamic Geometry software. The motivations for its development are presented as well as a detailed description of each part that makes up the MultiTela. The resource is developed from the study of Registers of Semiotics Representation, important for the learning process in mathematics theoretical foundation, as well as the prospect of adequately exploring the Semiotic Potential of Dynamic Geometry software. Have highlighted the implications of these theoretical development of MultiTela GD contributions. A brief description of their implementation ongoing training of mathematics teachers in distance education, and its reception by the participants is presented.
\end{abstract}

Keywords: MultiTela; dynamic geometry; distance education; mathematics teachers.

\section{Introdução}

O desenvolvimento das tecnologias digitais tem contribuído de forma substancial em diversas áreas de conhecimento, e também repercute no contexto escolar. Mais especificamente, no que se refere ao processo de ensino e aprendizagem de matemática, temos observado o desenvolvimento de diversos softwares específicos para seu ensino, a proliferação de materiais digitais, objetos de aprendizagem, sites especializados com videoaulas, e uma gama cada vez maior de aplicativos para dispositivos móveis. No entanto, a partir da análise de diversos estudos internacionais, Joubert (2013) destaca que as tecnologias digitais continuam sendo utilizadas de forma insuficiente nas aulas de matemática, e quando utilizadas, seu potencial ainda é pouco 
explorado. A partir destes fatores, a autora afirma que apenas disponibilizar recursos tecnológicos para escola não é suficiente, e que o ponto crucial é a análise do papel do professor no contexto escolar tecnológico, de modo que a formação de professores para o uso da tecnologia possui significativa importância.

Além disso, professores de todo país anseiam por formação complementar e continuada em cursos que sejam compatíveis com seu horário de trabalho, bem como não exijam grandes deslocamentos para centros de formação distantes geograficamente, o que também dificultaria a continuidade de sua atuação profissional. Nesse contexto, os cursos oferecidos na modalidade de Educação a Distância (EAD) são de extrema relevância para esta formação, permitindo que os participantes interajam em lugares e tempos diversos.

Tendo caracterizada a importância do professor no processo de incorporação das tecnologias digitais nas aulas de matemática, bem como da necessidade de formação básica ou complementar que seja compatível com seu horário e local de trabalho, os cursos oferecidos via EAD passam a ter cada vez mais importância neste contexto. Ao mesmo tempo, a modalidade EAD possui características específicas que implicam em um perfil específico dos participantes (PALLOFF; PRATT, 2004) para que o curso tenha sucesso.

E é dentro deste contexto que surge a MultiTela GD. A partir da análise e observações realizada em curso de especialização via EAD (Curso de Especialização em Matemática, Mídias Digitais e Didática para a Educação Básica - UFRGS/UAB), constata-se a pouca familiaridade do público alvo com esta modalidade, bem como com os recursos dos softwares de Geometria Dinâmica (GD) além das dificuldades com os conteúdos matemáticos e didáticos envolvidos.

Deste modo, a MultiTela GD foi desenvolvida para atender a formação de professores de matemática da educação básica para o uso de softwares de GD, mais especificamente o GeoGebra, no contexto da disciplina de Mídias Digitais I do curso indicado acima. A pouca familiaridade do público-alvo com o GeoGebra e a modalidade EAD, determinou o desenho de material didático digital que contribuísse para um processo de formação mais efetivo.

O recurso tem o objetivo de reduzir a dificuldade de visualização das diferentes telas no computador (GeoGebra, Moodle, website, applet, editor de texto, etc), que se tornam exaustivas e tiram a concentração do aprendizado. Analisando a formação de professores de matemática, Santos (2006, p. 102) observa que "maximizar/minimizar diferentes 'janelas' na tela do computador [...] se tornam ações constantes" e cansativas, e que dificultam a coordenação de todos os recursos. E é nesta questão que a MultiTela GD visa contribuir.

Além disso, a formação de professores de matemática para o uso de tecnologias requer o desenvolvimento de competências que articulem o potencial do recurso tecnológico com o conteúdo matemático que se deseja ensinar, e grande é a demanda cognitiva neste processo, o que justifica as escolhas de implementação da Multitela GD.

A seção 2 é destinada à uma apresentação detalhada de cada uma das partes que compõe a MultiTela GD, e a forma como o recurso foi desenvolvido. Na seção 3 é apresentado o aporte teórico que serviu de base para elaboração deste recurso, e como cada parte do recurso se articula com a teoria utilizada. A seção 4 traz um breve relato de sua implementação prática, e algumas percepções iniciais dos participantes do curso, e a conclusão e considerações finais são apresentadas na última seção. 


\section{Apresentando a MultiTela GD}

A MultiTela GD pode ser caracterizada como um multi-objeto de aprendizagem, tanto por integrar diferentes mídias quanto pela sua fácil adaptação para as mais diferentes representações em GD, de modo que é possível customizá-la para as mais diferentes atividades envolvendo o conteúdo de geometria.

O diferencial deste recurso não está na tecnologia empregada, mas na forma de organizar e disponibilizar o material digital desenvolvido, visando um processo didático que potencialize tanto a apropriação do software de GD quanto a compreensão dos conceitos matemáticos que estão sendo abordados.

De maneira geral pode se caracterizado como uma simples página web, desenvolvida em html e JavaScript, com quatro células que apresentam os materiais desenvolvidos. A figura 1 apresenta uma das MultiTelas GD desenvolvidas, que podem ser encontradas no item Recursos de cada módulo da disciplina Mídias Digitais I, disponível em http://www.ufrgs.br/espmat/disciplinas/midias_digitais_I/.

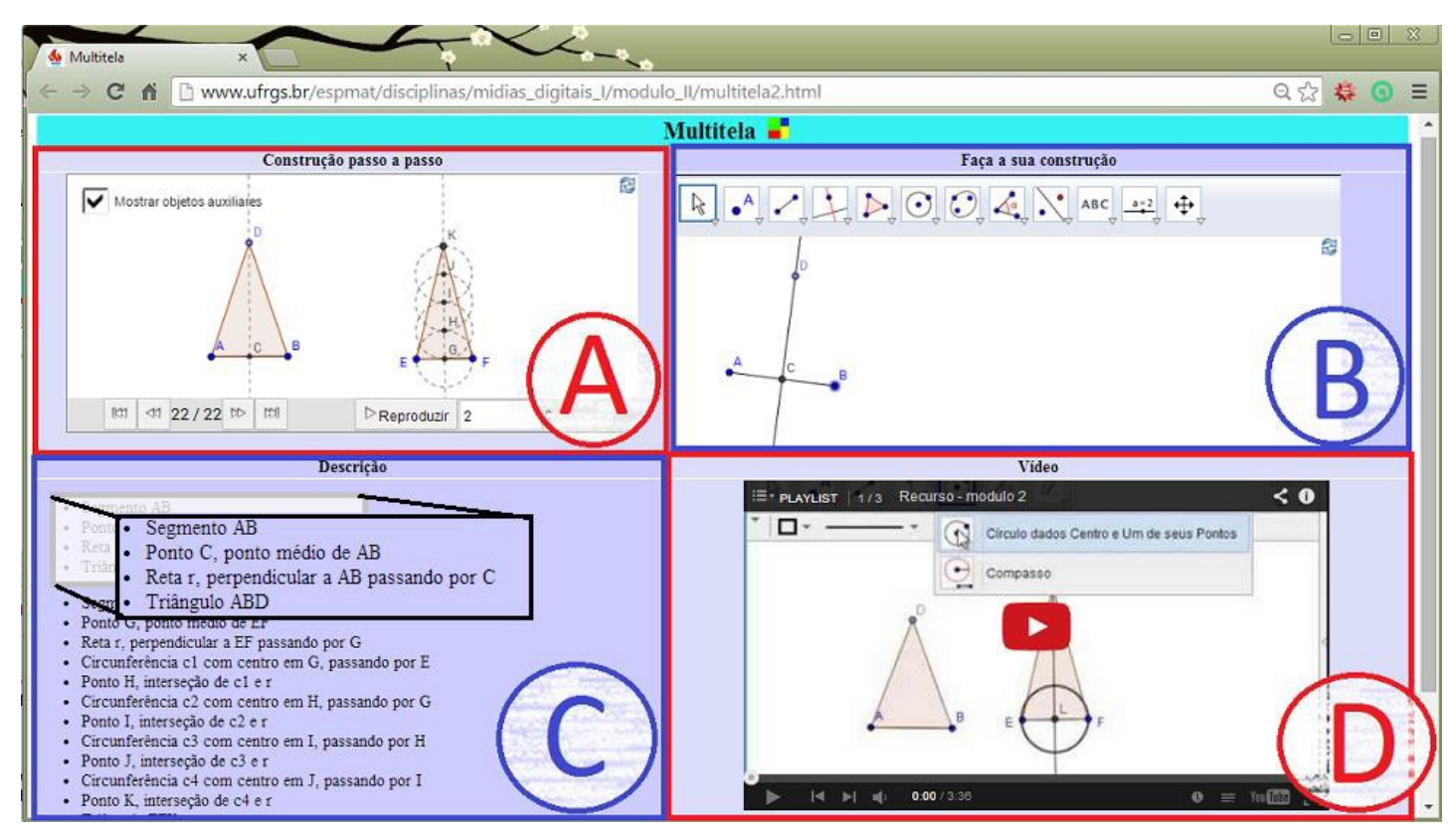

Figura 1: MultiTela GD.

A MultiTela GD conta com uma tabela com quatro células, assim organizadas:

- célula A - tem-se animação interativa (applet do GeoGebra) de uma construção geométrica. Esta construção é feita no software GeoGebra, exportada para o GeoGebraTube (http://www.geogebratube.org/), e em seguida incorporada à página web com código JavaScript. Nesta célula o usuário pode avançar, recuar, ou parar no procedimento de acordo com seu ritmo de aprendizagem. Ele também pode manipular os elementos da construção apresentada, analisando os invariantes geométricos presentes para elaborar conjecturas ou identificar as propriedades da construção. A Multitela GD apresentada na Figura 1 trata da construção de dois triângulos isósceles com propriedades distintas e portanto necessitam de construções diferentes no GeoGebra. 
- célula B - tem-se a tela do software GeoGebra incorporada na página web. Nela, o usuário pode realizar a construção geométrica, concomitante a visualização de cada etapa detalhada nas outras células. É importante destacar que o elaborador da MultiTela pode definir quais as ferramentas do software GeoGebra que estarão disponíveis aos usuários nesta célula, de acordo com os objetivos pedagógicos planejados para cada atividade.

- célula C - tem-se a descrição textual da construção proposta na animação, utilizando linguagem matemática pertinente.

- célula D - tem-se vídeo capturado na tela do GeoGebra; o vídeo apresenta a construção passo a passo, comentando cada ferramenta do GeoGebra utilizada, bem como esclarecendo e detalhando conceitos geométricos presentes na construção. Estes vídeos foram exportados para o YouTube (https://www.youtube.com/) e em seguida incorporados na página web. Deste modo, é possível editar os vídeos com hiperlinks remetendo para outros vídeos, de acordo com o interesse do usuário, criando assim hipervídeos e playlists de vídeos tratando do mesmo assunto.

Todas as células da MultiTela possuem foco na mesma construção apresentada na animação interativa apresentada na célula $A$, de modo que cada MultiTela GD elaborada apresenta uma construção ou atividade diferente.

O desenvolvimento da MultiTela GD com articulação de material adequado, rico em atividades e exemplos, com interação, pretende contribuir com o desenvolvimento de material mais autônomo para a EAD, no sentido de que menos intervenções não planejadas sejam necessárias para promover a aprendizagem.

Diferentes MultiTelas podem ser criadas, e apresentadas gradativamente aos usuários, tanto no que se refere aos conceitos matemáticos envolvidos quanto no uso adequado do software GeoGebra.

\section{Aporte teórico que norteou o desenvolvimento}

Em uma primeira impressão, percebe-se que a MultiTela GD apresenta diferentes recursos simultaneamente, sem a necessidade de maximizar/minimizar janelas, reduzindo o esforço cognitivo necessário para memorizar etapas de construção ou conceitos matemáticos apresentados nas janelas que eventualmente estivessem minimizadas. Ao mesmo tempo, em um único espaço é possível organizar diversos materiais abordando o mesmo conceito, possibilitando uma visão mais abrangente sobre o conteúdo disponibilizado naquela multitela.

No entanto, a motivação do seu desenvolvimento vai muito além desta questão 'prática', e está alicerçada em três aportes teóricos diferentes, que serão apresentados brevemente:

- Abordagem Instrumental

- Registros de Representação Semiótica

- Potencial Semiótico

A Abordagem Instrumental tem origem na Ergonomia Cognitiva com autores como Rabardel (1995) e outros, tendo sua atenção central no uso de ferramentas pelo ser 
humano. De forma mais específica, as ferramentas (tais como martelo, lápis, software, etc.) inicialmente são chamados de artefatos, e somente passam a ser nomeados de instrumento quando a eles forem associados esquemas de utilização por parte do ser humano.

Esta passagem de artefato para instrumento, quando o indivíduo vai associando gradativamente esquemas de utilização ao artefato, é denominado de Gênese Instrumental (Artigue, 2002) e não é um processo simples e nem rápido.

Neste sentido, a MultiTela GD visa a contribuir com a formação de professores de matemática via EAD, possibilitando que cada usuário possa identificar gradativamente ferramentas do GeoGebra, e atribuindo a ele esquemas de utilização, transformando efetivamente em um instrumento.

É preciso destacar que a Gênese Instrumental não se resume a instruir como funciona cada ferramenta do GeoGebra, mas um processo longo e demorado, em que o GeoGebra vai sendo apropriado pelo usuário, até chegar ao ponto, por exemplo, em que qualquer problema de geometria passa a ser pensado automaticamente com o uso do GeoGebra, sem que haja qualquer indicação para isso (Bittar, 2011). Ou seja, o usuário incorpora o GeoGebra na forma com que ele pensa matemática.

E como a MultiTela GD contribui para o processo de Gênese Instrumental de cada indivíduo na apropriação do GeoGebra em cursos EAD? De diversas formas. No contexto geral, apresentando diferentes MultiTelas para desenvolver os esquemas de utilização de forma gradativa e consistente.

Além disso, cada célula possui contribuições específicas no processo de Gênese Instrumental:

- A: a possibilidade de movimento e interação com o applet permite que o usuário identifique quais os recursos do GeoGebra que foram utilizados para fazer aquela construção, e em que sequência foram feitos, avançando ou retrocedendo na construção passo a passo.

- C: os elementos construídos estão descritos sequencialmente, permitindo que se diferencie uma representação geométrica de uma reta na célula A, se ela é uma reta perpendicular, paralela, por um ponto de interseção, etc. Ou seja, a célula $\mathrm{C}$ complementa a célula $\mathrm{A}$, indicando com precisão o elemento representado, facilitando a identificação da ferramenta correta a ser utilizada no GeoGebra para fazê-lo.

- B: é o espaço para colocar em prática o que foi observando nas outras células, e efetivamente desenvolver seus próprios esquemas de utilização de cada ferramenta do software; também permite que se faça o uso de tentativa e erro, verificando se a construção obtida possui características semelhantes às apresentadas na célula $\mathrm{A}$, e descritas na célula $\mathrm{C}$.

- D: é a célula que permite o acompanhamento comentado da construção, esclarecendo dúvidas sobre a forma correta de usar determinada ferramenta, bem como das implicações na construção quando determinada ferramenta é utilizada ou não.

Outro aporte teórico importante é o dos Registros de Representação Semiótica (Duval, 2003; 2009), dado que qualquer os elementos matemáticos (reta, ponto, circunferência) são abstratos, e somente podem ser acessados através da representação por signos (neste texto signo é entendido como a união indissolúvel entre significado e 
significante). Desta forma, a representação semiótica é primordial à qualquer atividade matemática.

Segundo Duval (2009, p.15), em matemática “não há noesis sem semiosis’, ou seja não é possível compreender determinado conceito sem a utilização de signos que o representem. $\mathrm{O}$ autor classifica os signos em diferentes registros ou sistemas: discursivo; figural, algébrico e numérico; e gráfico.

A partir destes diferentes registros, as mudanças de representação (da representação algébrica para a gráfica, por exemplo) são centrais na matemática, já que cada representação é parcial em relação ao conteúdo do objeto, e a utilização de diferentes registros para representar o mesmo elemento, bem como a "a articulação dos registros é condição de acesso à compreensão em matemática” (Duval, 2003).

Na MultiTela GD, os diferentes registros de representação ficam evidentes em cada célula. Na primeira há o registro figural do ente geométrico, onde também poderá ser utilizado o registro numérico (quando associamos medidas aos elementos geométricos, por exemplo). Na célula $\mathrm{C}$ temos o registro discursivo, e se considerarmos a passagem $\mathrm{A}->\mathrm{C}$, temos a mudança do registro figural para o discursivo, e o inverso acontece em $\mathrm{C}->\mathrm{A}$ ou $\mathrm{C}->\mathrm{B}$, já que em $\mathrm{B}$ o usuário terá que utilizar a informação discursiva e transformá-la em representação geométrica figural. Na célula $\mathrm{D}$, além da possibilidade de articular qualquer um dos registros nos vídeos, há o destaque para os comentários sobre os conceitos matemáticos envolvidos, o que em diversas vezes facilita a compreensão ou a identificação de propriedades subjacentes, e principalmente permite ao professor comentar o que acontece na mudança de registros, facilitando a compreensão por parte do usuário.

Dada a importância da utilização de diferentes registros semióticos para a compreensão e aprendizagem em matemática, a Multitela contribui significativamente nesta articulação, por apresentar diferentes registros simultaneamente, bem como incentiva a mudança de um registro para outro, atuando de forma a fomentar a compreensão dos conceitos pelo usuário.

O terceiro aporte teórico, Potencial Semiótico, surge da confluência dos dois aportes apresentados anteriormente, e que são identificáveis nos softwares de GD. Segundo Bussi e Mariotti (2008) e Mariotti (2012), Potencial Semiótico é a potencialidade do artefato (neste caso o GeoGebra) articular significados pessoais que emergem das atividades instrumentadas (atividades com o instrumento, conforme a Gênese Instrumental) e os significados matemáticos através dos diferentes registros contidos no software ou articulados pelo professor nas atividades.

Considerando a formação de professores de matemática para o uso de tecnologia, segundo Stormowski, Gravina e Lima (2013) é essencial a identificação do Potencial Semiótico dos softwares de GD, dado que segundo Mariotti (2009) esta identificação é elemento básico para a concepção de plano pedagógico no uso de artefatos.

E a MultiTela GD foi planejada de forma a possibilitar a identificação do Potencial Semiótico do GeoGebra por parte do usuário. Cada uma das células apresenta conceitos matemáticos representados de diversas formas, e cada um destes conceitos está diretamente relacionado a alguma atividade com o artefato GeoGebra, onde é necessário fazer construções geométricos (atividades com o artefato), mas que necessariamente precisam envolver conceitos matemáticos para garantir as propriedades identificadas em cada construção. 
Além disso, o próprio planejamento de cada atividade a ser apresentada pela MultiTela, já leva em consideração quais os conceitos matemáticos que se pretende mobilizar e através de quais ferramentas ou quais usos do GeoGebra que se pretende fazêlo, evidenciando assim o Potencial Semiótico do software.

\section{Breve relato da implementação}

A primeira implementação da MultiTela GD ocorreu na disciplina de Mídias Digitais I do curso de Especialização em Matemática, Mídias Digitais e Didática para a Educação Básica da UFRGS/UAB. Esta disciplina foi organizada em sete módulos, abordando concomitantemente conteúdos de matemática (geometria, álgebra e funções) e atividades de apropriação do software GeoGebra, combinados com debates (fóruns) a respeito das possibilidades de uso destes recursos tecnológicos em sala de aula. É importante destacar que a abordagem matemática e do uso do software não foi feita separadamente, mas tudo articulado através de uma mesma atividade, planejada e estruturada com o apoio das MultiTelas.

As MultiTelas desenvolvidas para a disciplina estão disponíveis no item Recursos de cada módulo em http://www.ufrgs.br/espmat/disciplinas/midias_digitais_I/. Ao todo foram desenvolvidas dez MultiTelas diferentes para contemplar cada um dos módulos da disciplina. Dependendo do planejamento de cada módulo, foram elaboradas uma determinada quantidade de MultiTelas que atendesse os objetivos estabelecidos.

A disciplina ocorreu de março a maio de 2014, contando com cerca de cento e cinquenta alunos distribuídos em seis polos presenciais distribuídos pelo estadão do Rio Grande do Sul.

Ao final da disciplina foi apresentada uma “Avaliação Geral da disciplina” com participação voluntária, de modo que 93 dos alunos responderam. Dentre os itens respondidos, alguns versavam sobre o uso da MultiTela GD. Dentre os itens, destacamos:

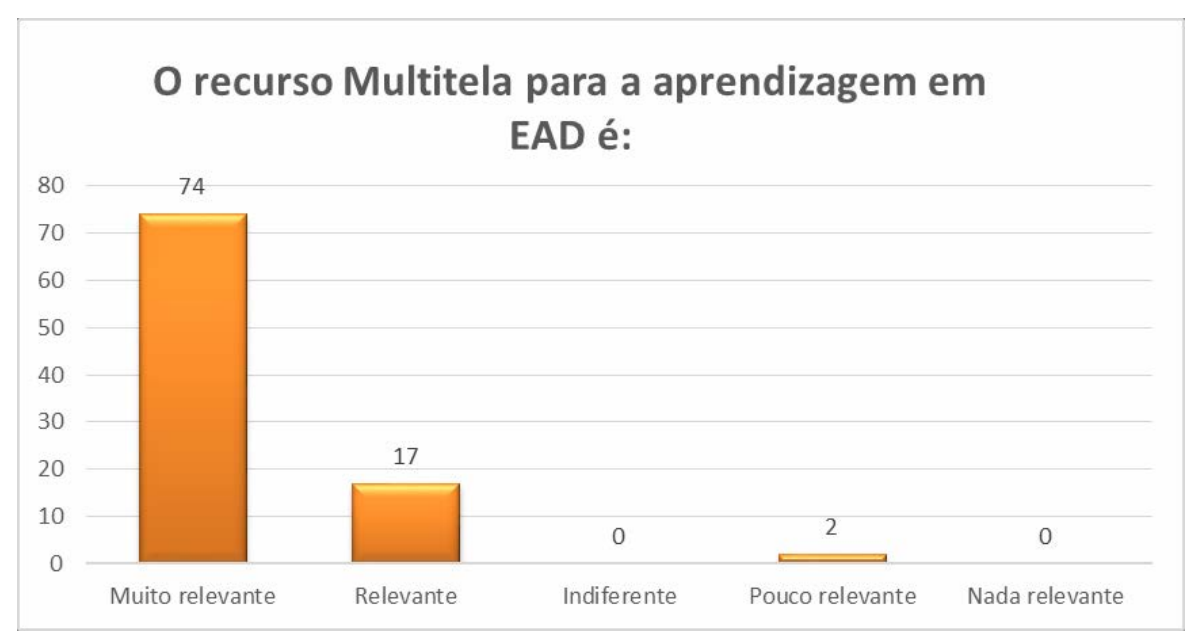

Figura 2: relevância da MultiTela GD para a EAD

Uma proporção expressiva (cerca de $80 \%$ ) das respostas confirma a satisfação dos usuários com o uso da MultiTela GD em cursos EAD, o que também é confirmado em diferentes repostas quando foram solicitados comentários sobre o recurso. Respostas como "foi um plus neste material, quando me sentia insegura quanto ao que estava sendo apresentado me encontrava por ali e claro no material de apoio", "É um espaço ótimo, 
pois permite testar, observar, verificar, tudo em um só local... Parabéns pela ideia." e "É como uma aula...”, enfatizam a satisfação de boa parte dos usuários. Nesta última, parece ser uma clara alusão à aula presencial, dando a entender que o respondente considera o processo na modalidade EAD muito diferente do presencial.

A figura 3 apresenta o gráfico com os dados do questionário sobre os motivos que os respondentes disseram ter utilizado a MultiTela GD.

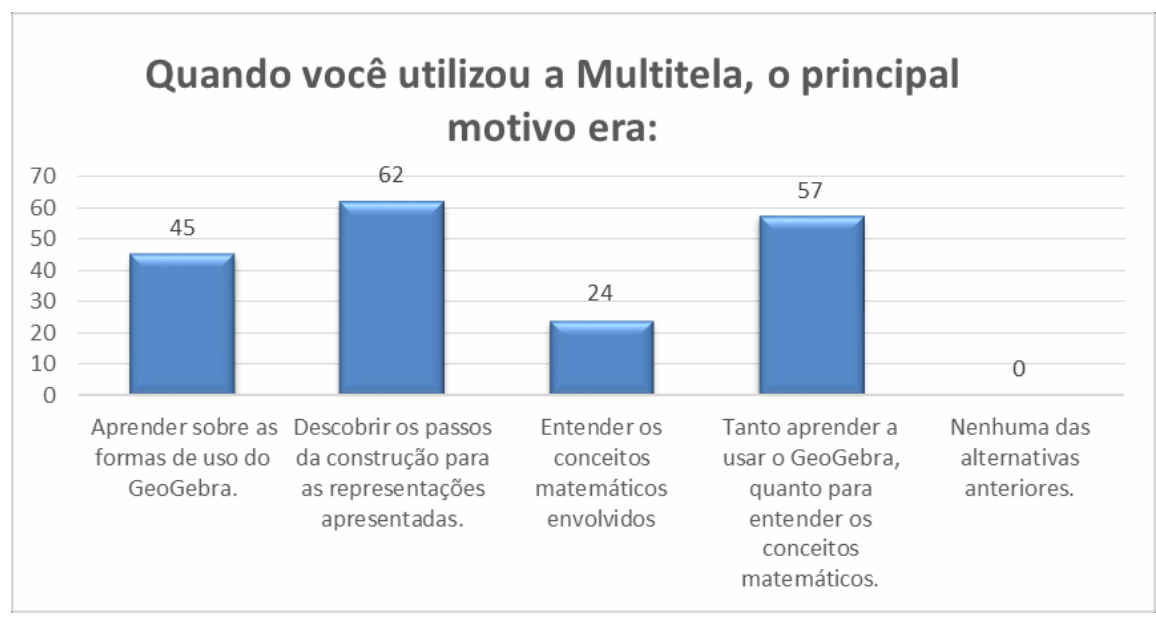

Figura 3: o motivo por usar a MultiTela GD

Era permitido marcar mais de uma alternativa, e independente disto, observa-se que há uma divisão entre os respondentes que dizem utilizar para aprender formas de uso do GeoGebra e os que dizem ter utilizado para entender conceitos matemáticos (em menor número), mas é interessante a proporção daqueles que dizem ter utilizado por ambos os motivos.

Na figura 4 temos os motivos da não utilização da MultiTela.

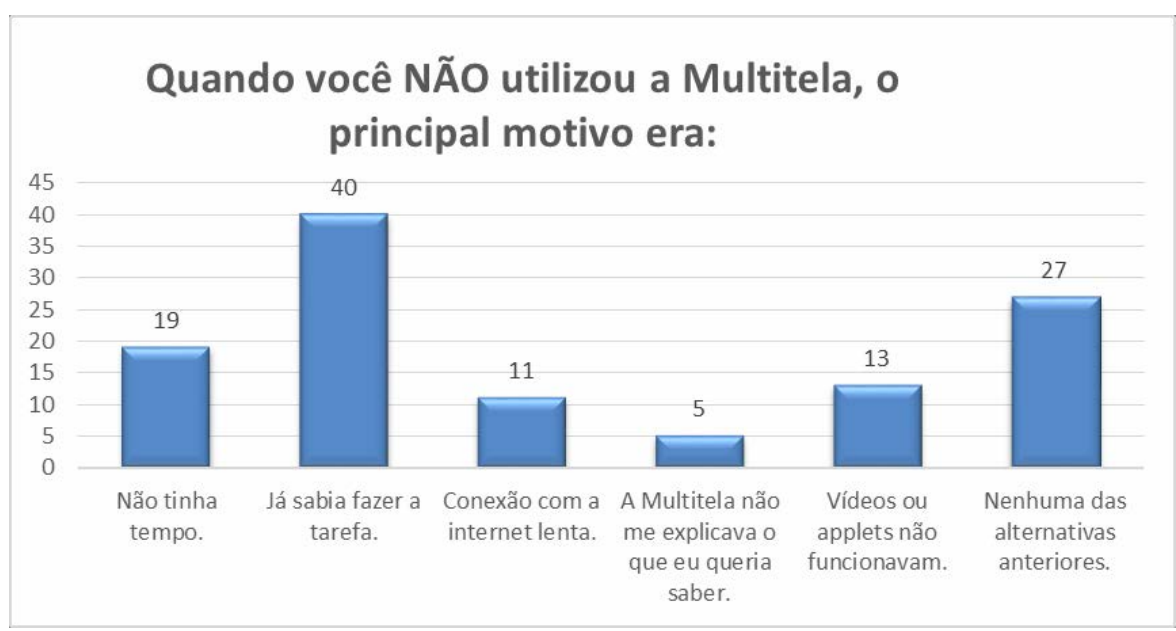

Figura 4: o motivo por não usar a MultiTela GD

Se destacam aqueles que não utilizaram por já saberem fazer a tarefa, e os com falta de tempo (que parece estar relacionada com a atividade profissional de cada um, já que todos eram professores atuantes em escolas de Educação Básica). 
É importante observar também as dificuldades técnicas, tais como o não funcionamento de vídeos ou applets, que na maioria das vezes está relacionada ao tipo de conexão com a internet, que dificulta a transferência de vídeos e outros applets. Estes dados são muito importantes para o futuro aperfeiçoamento do recurso, com possibilidade novas implementações em breve.

\section{Considerações finais}

A MultiTela GD nasceu de uma solução técnica simples, mas que está fortemente relacionada com o aporte teórico apresentado, que faz parte de um estudo maior em andamento. É com profunda reflexão sobre a prática existente, amparada com aporte teórico consistente, que possibilitou o desenvolvimento deste recurso, tendo destaque quando nos referimos à modalidade EAD de formação e professores de matemática.

O feedback dos usuários permite que o recurso continue sendo aprimorado, possibilitando melhorias para futuras implementações. Ao mesmo tempo, a MultiTela GD obteve muitas observações de avaliadores técnicos, quando concorreu ao Prêmio UniRede de Inovação Tecnológica realizado no Congresso Brasileiro de Ensino Superior a Distância - ESUD 2014, obtendo $2^{\circ}$ lugar dos avaliadores técnicos e $4^{\circ}$ lugar considerando também a votação do público (Unirede, 2014). Os diferentes pareceres dos avaliadores técnicos também são indicativos de melhorias que estão sendo realizadas.

Além de tudo, é preciso destacar a simplicidade técnica do recurso, já que envolve apenas html e JavaScript simples, podendo ser editado e configurado da forma com que cada professor de cursos EAD desejar, pois permite a exploração das mais diferentes construções geométricas.

\section{Referências}

ARTIGUE, M. Learning mathematics in a CAS environment: the Genesis of a reflection about instrumentation and the dialectics between technical and conceptual work. International Journal of Computers for Mathematical Learning, Netherlands, n. 7. p. 245-274, 2002.

BITTAR, M. A abordagem instrumental para o estudo da integração da tecnologia na prática pedagógica do professor de matemática. Educar em revista, Curitiba, p. 157-171, 2011.

BUSSI, M. G. B.; MARIOTTI, M. A. Semiotic mediation in the mathematics classroom: artifacts and signs after a vygotskian perspective. Handbook of international research in mathematics education, New York, p. 746-783, 2008.

DUVAL, R. Registros de representações semióticas e funcionamento cognitivo da compreensão em matemática. In: Aprendizagem em matemática: registros de representação semiótica. Campinas: Papirus, 2003. p. 11-34.

DUVAL, R. Semiósis e Pensamento Humano: registros semióticos e aprendizagens intelectuais. São Paulo: Livraria da Física, 2009.

JOUBERT, M. Using digital technologies in mathematics teaching: developing an understanding of the landscape using three "grand challenge" themes. Educational Studies in Mathematics, vol. 82, n. 3, p. 341-359, mar. 2013. 
MARIOTTI, M. A. Artifacts and signs after a Vygotskian perspective: the role of the teacher. ZDM Mathematics Education, vol. 41, p. 427-440, 2009.

MARIOTTI, M. A. ICT as opportunities software for teaching-learning in mathematics classroom: the semiotic potential of artefacts. In: TSO, T. Y. (Org.). Proceedings of the $36^{\text {th }}$ Conference of the International Group for the Psychology of Mathematics Education. Taipei, Taiwan: PME, 2012. vol. 1, p. 25.

PALLOFF, R. M.; PRATT, K. O aluno virtual: um guia para trabalhar com estudantes on-line. Porto Alegre: Artmed, 2004.

RABARDEL, P. Les hommes et les technologies: aprproche cognitive des instruments contemporains. Paris: Armand Colin, 1995.

SANTOS, S. C. A produção matemática em um ambiente virtual de aprendizagem: o caso da geometria euclidiana espacial. 2006. Dissertação de Mestrado em Educação Matemática - UNESP, Rio Claro.

STORMOWSKI, V.; GRAVINA, M. A.; LIMA, J. V. Tecnologia na aula de matemática: a importância do potencial semiótico. RENOTE - Revista Novas Tecnologias na Educação, Porto Alegre, vol.11, n. 3, Dezembro 2013.

UNIREDE. Resultado do Prêmio Unirede de Inovação Tecnológica 2014. Disponível em: $<$ http://esud2014.nute.ufsc.br/wp-content/uploads/2014/09/ResultadoPr\%C3\%AAmio-UniRede.pdf > . Acesso em: 28 dez. 2014. 